\title{
Effect of martensitic phase transformation on the behavior of 304 austenitic stainless steel under tension
}

\author{
H. Wang, ${ }^{\mathrm{a},}$, Y. Jeong ${ }^{\mathrm{b}}$, B. Clausen ${ }^{\mathrm{a}}$, Y. Liu ${ }^{\mathrm{a}}$, R.J. Mccabe ${ }^{\mathrm{a}}$, F. Barlat ${ }^{\mathrm{c}}$, C.N. Tomé ${ }^{\mathrm{a}}$ \\ aMaterials Science and Technology, Los Alamos National Laboratory, Los Alamos, NM, USA \\ bMaterials Science and Engineering Division, National Institute of Standards and Technology, \\ Gaithersburg, MD, USA \\ ${ }^{\mathrm{c}}$ Graduate Institute of Ferrous Technology, POSTECH, Korea
}

\begin{abstract}
The present work integrates in-situ neutron diffraction, electron backscatter diffraction and crystal plasticity modeling to investigate the effect of martensitic phase transformation on the behavior of 304 stainless steel under uniaxial tension. The macroscopic stress strain response, evolution of the martensitic phase fraction, texture evolution of each individual phase, and internal elastic strains were measured at room temperature and at $75^{\circ} \mathrm{C}$. Because no martensitic transformation was observed at $75^{\circ} \mathrm{C}$, the experimental results at $75^{\circ} \mathrm{C}$ were used as a reference to quantify the effect of formed martensitic phase on the behavior of 304 stainless steel at room temperature. A crystallographic phase transformation model was implemented into an elasticviscoplastic self-consistent framework. The phase transformation model captured the macroscopic stress strain response, plus the texture and volume fraction evolution of austenite and martensite. The model also predicts the internal elastic strain evolution with loading in the austenite, but not in the martensite. The results of this work highlight the mechanisms that control phase transformation and the sensitivity of modeling results to them, and point out to critical elements that still need to be incorporated into crystallographic phase transformation models to accurately describe the internal strain evolution during phase transformation.
\end{abstract}

Keywords: Transformation induced plasticity (TRIP) steel; Crystal plasticity; in-situ neutron diffraction; EBSD

\footnotetext{
${ }^{*}$ Corresponding author. Dr. Huamiao Wang. Tel.: 1-505-664-0321, Email: wanghm@lanl.gov.
} 


\section{Introduction}

TRIP (transformation induced plasticity) steels are characterized by their excellent combination of strength, ductility and response to high-speed deformation and are thus extensively used in the automobile industry [1-3]. In addition to common strengthening mechanisms, such as grain refinement, precipitation or composite strengthening both, strength and ductility, can be improved by the contribution of martensitic phase transformation [4,5]. A lot of works have investigated the microscopic plastic behavior of TRIP steels using techniques of Transmission Electron Microscopy, Electron Backscatter Diffraction (EBSD), and Neutron Diffraction, etc. $[3,6,7]$. Among the measuring techniques, neutron diffraction is well adapted for the characterization of the microscopic plastic behavior of TRIP steels because of its selectivity based on the crystal lattices and the large size of gauge volume. Moreover, in-situ neutron diffraction measurement provides separate information about the evolution of internal elastic strains (or internal stress) for each phase of the TRIP steel under deformation. The transformed martensitic phase results from the combination of shear and dilatational volume expansion, which in return induces additional plasticity in the surrounding matrix by imposing locally concentrated stress field [8-10]. The precise measurement of the lattice strains under such a circumstance will lead us to a better understanding of the TRIP effect. Several studies have demonstrated the possibility of monitoring the stress partitioning between the austenitic and martensitic phases using in-situ neutron diffraction during mechanical straining [6,7,11].

Most of the in-situ neutron measurements on TRIP effect have been performed to relatively small plastic strains. In addition, the data in those measurements were collected through periodically interrupting the loading while holding either the stress or the strain constant. In such a case, the holding times could be as long as 45 minutes [12]. As a consequence, either stress or strain relaxation takes place during data collection, with the inconvenience that the internal strains evolve during the measurement. An alternative testing technique, which consists in performing in-situ measurements on specimens deformed uninterruptedly at very low strain rates $\left(10^{-6}-10^{-5} \mathrm{~s}^{-1}\right)$, was recently applied to austenitic steel by An et al. [13] and Wang et al [14]. This technique has the advantage over the conventional measuring techniques that it avoids the stress or strain relaxation associated with the interrupts during lattice strain measurements. 
An accurate constitutive model is demanded to interpret shifts of diffraction peaks in terms of internal strain pertaining to a specific subset of grains. Various constitutive models for martensitic phase transformation have been proposed in the literature [9,10,15-21]. These models fall into two categories: phenomenological and crystallographic mechanisms-based models. None of them, however, addresses the probabilistic relations between the microstructural/stress variability and phase transformation variant selection. Modeling the evolution of internal elastic strain under loading becomes even more challenging when phase transformation is present, and requires incorporating explicitly lattice scale mechanisms.

We pursue experimental and modeling goals in the present work. The first goal is to apply the uninterrupted in-situ neutron diffraction measuring technique to study the influence of martensitic phase transformation on the large deformation behavior of TRIP steel. Two tension tests of 304 austenitic stainless steel at 25 and $75{ }^{\circ} \mathrm{C}$ are performed. Since phase transformation takes place at room temperature, but does not at $75^{\circ} \mathrm{C}$ for the steel tested here, the test at $75{ }^{\circ} \mathrm{C}$ is then used as a baseline to evaluate the influence of martensitic transformation on the behavior of 304 austenitic stainless steel both qualitatively and quantitatively. The second goal is to develop a crystallographic mechanisms-based model for martensitic phase transformation with mechanical predictive capabilities. Such a model should account for various modeling elements that include: a) a nucleation and variant selection criterion; b) the introduction of martensitic grains in the aggregate; c) the orientation relationship between the martensitic grain and its parent austenitic phase; d) the evolution of the martensitic grain and the implementation of the martensitic phase transformation strain. In this work, those modeling elements are incorporated into the elastic viscoplastic self-consistent (EVPSC) model [14,22-26]. The EVPSC model has been successfully applied to study the evolution of internal elastic strain of stainless steel not exhibiting transformation [14,27], magnesium alloys [28-30] and zirconium alloys [31]. The polycrystal model is applied to interpret the hardening, texture and phase evolution during the insitu neutron diffraction measurement on the 304 stainless steel. And the model is applied to interpret for the first time the evolution of diffraction peak intensity and internal elastic strain in both austenitic and martensitic phases as they evolve. Section 2 of this paper presents a description of the experimental procedure. Section 3 presents a detailed description of the specific elements added to the EVPSC model. The experimental and simulated results are compared and discussed in Section 4. 


\section{Experimental procedure}

A 304 austenitic stainless steel sheet with average grain size of $25 \mu \mathrm{m}$ was investigated. The chemical composition is listed in Table 1. The microstructures were characterized by Electron Backscatter Diffraction (EBSD) analysis. A pure austenitic phase was observed in the EBSD orientation map of the undeformed sample (Fig. 1a). Dog-bone tension specimes were machined such that the loading axis aligns with the rolling direction (RD) of the stainless steel sheet. The EBSD orientation map at 30\% tensile strain is presented in Fig. 1b, where it can be seen that mainly one martensite variant was activated in most of the grains. The latter observation is relevant to the modeling assumptions done in this work. The spectrometer for high intensity pressure and preferred orientation (HIPPO) at LANSCE (Los Alamos Neutron Science Center) was used to measure the textures. Fig. 2 shows the $\{111\},\{200\}$ and $\{220\}$ pole figures of the initial austenitic phase. The pole figures indicate that the as-received stainless steel has a very weak rolling texture. The textures at tensile strains of $10 \%, 20 \%, 30 \%$ and $40 \%$ were also measured and will be reported in Section 4.

In-situ neutron diffraction measurements were performed during tensile deformation using the Spectrometer for Materials Research at Temperature and Stress (SMARTS), also at LANSCE (Details of the instrument can be found in Bourke et al. [32]). Uniaxial tension tests at a strain rate of $10^{-5} / \mathrm{s}$ were performed at two different temperatures, i.e., room temperature (RT) and $75^{\circ} \mathrm{C}$. An induction coil was used to heat the sample to the specified temperature. This low strain rate avoids a temperature increase induced by deformation. Within such a narrow temperature interval, the properties of austenitic phase are not subject to change significantly. Martensitic phase is transformed from austenitic phase under tension at RT, while it is not observed at $75^{\circ} \mathrm{C}$. Therefore the effect of martensitic phase transformation is investigated through comparing the two deformation behaviors of stainless steel at RT and at $75^{\circ} \mathrm{C}$. The load frame is oriented at a $45^{\circ}$ angle to the incident beam and thus the two detector banks at $\pm 90^{\circ}$ to the incident beam allow for simultaneous measurement of diffraction patterns with scattering vectors parallel and transverse to the loading axis, respectively. The neutron data are collected continuously throughout all the testing time using the uninterrupted data acquisition format [14], and the time-of-flight technique measures the full diffraction profile in each diffraction bank. 
Internal elastic strains are calculated from changes in peak position during deformation through the equation $\varepsilon^{h k l}=\left(d^{h k l}-d_{0}^{h k l}\right) / d_{0}^{h k l}$. The 'stress-free' reference lattice spacing for each peak,

$d_{0}^{h k l}$, was taken as the measured lattice spacing in the austenitic phase prior to applying any load to the sample. The absence of martensitic phase at the onset of the experiment means that there is no such reference value to calculate the internal elastic strain pertaining to the martensitic phase. This is an important issue for interpreting the measurements. Our approach consists on calculating a stress-free lattice constant for martensitic phase from the stress-free austenite lattice constant and the martensite/austenite density ratio [33],

$$
\frac{a_{\text {mart }}}{a_{\text {aust }}}=\sqrt[3]{\frac{\rho_{\text {aust }}}{\rho_{\text {mart }}}}
$$

where $a_{\text {mart }}$ and $a_{\text {aust }}$ are the lattice parameters for martensite and austenite, respectively. $\rho_{\text {aust }}$ and $\rho_{\text {mart }}$ are the density of austenite and martensite, respectively. The density data of martensite and austenite for multi component steels are given by the following expressions:

$$
\begin{array}{rl}
\rho_{\text {mart }}=7875 & 96-0.297 T-5.62 \times 10^{-5} T^{2} \\
& +\left(-206.35+0.00778 T+1.472 \times 10^{-6} T^{2}\right) C_{C}^{\text {mart }} \\
& +\left(-8.58+1.229 \times 10^{-3} T-0.852 \times 10^{-7} T^{2}+0.018367 C_{C r}^{\text {mart }}\right) C_{C r}^{\text {mart }} \\
& +\left(-0.22-0.470 \times 10^{-3} T-1.855 \times 10^{-7} T^{2}-0.104608 C_{N i}^{\text {mart }}\right) C_{N i}^{\text {mart }} \\
& -36.86 C_{S i}^{\text {mart }}-7.24 C_{M n}^{\text {mart }}+30.78 C_{M o}^{\text {mart }} \\
\rho_{\text {aust }}=8099.79-0.506 T+\left(-118.26+7.39 \times 10^{-3} T\right) C_{C}^{\text {aust }} \\
& +\left(-7.59+3.422 \times 10^{-3} T-5.388 \times 10^{-7} T^{2}-0.014271 C_{C r}^{\text {aust }}\right) C_{C r}^{\text {aust }} \\
& +\left(1.54-2.267 \times 10^{-3} T-11.26 \times 10^{-7} T^{2}+0.062642 C_{N i}^{\text {aust }}\right) C_{N i}^{\text {aust }} \\
& -68.24 C_{S i}^{\text {aust }}-6.01 C_{M n}^{\text {aust }}+12.45 C_{M o}^{\text {aust }}
\end{array}
$$

where $C_{i}$ is solute content in wt $\%, T$ is temperature in ${ }^{\circ} \mathrm{C}$, and units are $\mathrm{kg} \cdot \mathrm{m}^{-3}$. The solute content of each element is listed in Table 1.

\section{Model description}

\subsection{Phase Transformation (PT) model}

Four aspects of martensitic transformation need to be addressed for modeling purposes: a) a criterion for martensitic variant selection in the austenitic phase; b) introduction of the newly formed martensitic phase in the aggregate, including the initial internal stress; c) volumetric 
evolution of martensitic phase; d) implementation of the martensitic phase transformation strain (MPTS).

\section{a) Variant selection}

There are 24 possible crystallographic variants associated with the martensitic phase transformation but, usually, only a few of the available variants are activated in a given grain under deformation [34]. As mentioned in Introduction, our EBSD orientation map (Fig. 1b) at 30\% strain indicates that mostly only one variant is activated in each austenite grain, therefore in our model we allow for only one variant per grain. An energy-based variant selection criterion is employed in this work. For the $I^{\text {th }}$ variant, the energy $E^{\mathrm{I}}(\mathrm{I}=1,2, \cdots, 24)$ associated with the phase transformation is estimated using the product of the grain stress $\left(\boldsymbol{\sigma}^{\mathrm{g}}\right)$ and the corresponding martensitic phase transformation strain $\left(\boldsymbol{\varepsilon}_{I}^{\mathrm{PT}}\right.$, determination of the martensitic phase transformation strain is given later):

$$
E^{\mathrm{I}}=\boldsymbol{\sigma}^{\mathrm{g}}: \boldsymbol{\varepsilon}_{I}^{P T}
$$

Positive values of $E^{\mathrm{I}}$ indicate that the transformation is 'aided' by the stress state in the grain, rather than having to work 'against' it. And although not all the transformation is accommodated elastically, it is plausible that the elastic energies of the variants (Eq. 2) are in the same order as the total relaxation energies. Therefore, the variant $\left(I_{\max }\right)$ with the maximum positive dissipation $E_{\max }^{\mathrm{I}}$ is selected to be the candidate for phase transformation in the austenitic grain (g). The other martensitic variants are not allowed to transform in the corresponding grain from then on.

\section{b) Introduction of martensitic phase}

A martensitic phase associated with variant $I_{\max }$ is introduced when the Von Mises equivalent strain of the austenitic grain reaches a critical value $\left(\varepsilon_{c r}^{P T}\right)$. The introduced martensitic grain is treated as a new member in the polycrystalline aggregate with an initially small volume fraction (0.001 of the austenitic parent grain) and a flat ellipsoidal shape with 1:10 aspect ratio. The initial states of the newly nucleated martensitic grain, i.e. orientation, stress, morphology, and volume fraction, have to be assigned. According to the crystallographic theory of martensite, the orientation relationship between the austenite and martensite is irrational [35-37]. In certain 
cases the Kurdjumov-Sachs (K-S) [38] or Nishiyama-Wasserman (N-W) [39] relationship is often used. The difference among the irrational, the K-S and the N-W orientations is less than a few degrees. However K-S (or N-W) relationship does not lead to an invariant line between the parent and product lattices. The existence of an invariant line is an essential requirement for martensitic transformation to occur [35].

When describing martensitic transformation, the crystallographic set of habit plane, invariant line, shape deformation, martensitic phase transformation strain and orientation relationship should be considered geometrically as a whole [35-37]. According to Bhadeshia [40], the parameters associated with the crystallographic mechanisms associated with a martensitic phase transformation are functions of lattice parameters of austenite and martensite. As reported by Dyson and Holmes [41], the lattice parameters of austenite and martensite are respectively $0.3589 \mathrm{~nm}$ and $0.2873 \mathrm{~nm}$. Then the rotation matrix $\left(\boldsymbol{R}^{\mathrm{PT}}\right)$ that relates austenitic orientation to that of martensite is given by

$$
\boldsymbol{R}^{P T}=\left(\begin{array}{ccc}
0.7253 & -0.6881 & -0.0240 \\
0.6756 & 0.7179 & -0.1678 \\
0.1327 & 0.1055 & 0.9855
\end{array}\right)
$$

This rotation matrix transforms the coordinates of a material vector $\mathbf{u}$ expressed in crystal basis of austenite to one expressed in crystal basis of martensite. This rotation matrix should correlate the orientations between martensite and austenite by satisfying the equations:

$$
\left[\begin{array}{lll}
0.5774 & 0.5774 & 0.5774
\end{array}\right]_{\text {aust }}=\left[\begin{array}{lll}
0.0076 & 0.7077 & 0.7065
\end{array}\right]_{\text {mart }}
$$

and

$$
\left(\begin{array}{lll}
-0.7071 & 0 & 0.7071
\end{array}\right)_{\text {aust }}=\left(\begin{array}{lll}
-0.5298 & -0.5964 & 0.6030
\end{array}\right)_{\operatorname{mart}}
$$

This means that $\left[\begin{array}{lll}1 & 1 & 1\end{array}\right]_{\text {aust }}$ is nearly parallel to $\left[\begin{array}{lll}0 & 1 & 1\end{array}\right]_{\text {mart }}$ and $\left(\begin{array}{lll}1 & 0 & 1\end{array}\right)_{\text {aust }}$ is about $3^{\circ}$ from $(\overline{1} \overline{1} 1)_{\text {mart }}$, which is very close to the K-S orientation relationship.

The deformation gradient associated with the martensitic phase transformation is

$$
\boldsymbol{F}=\left(\begin{array}{ccc}
0.991 & -0.037 & -0.028 \\
0.030 & 1.125 & 0.095 \\
-0.028 & -0.119 & -0.909
\end{array}\right)
$$


The associated dilatational and shear strains are $3.7 \%$ and $22 \%$, respectively. The martensitic phase transformation strain $\boldsymbol{\varepsilon}^{\mathrm{PT}}$ is determined from the above deformation gradient (referred to austenitic axes)

$$
\varepsilon^{\mathrm{PT}}=\frac{\left(\boldsymbol{F}^{T} \cdot \boldsymbol{F}-\mathbf{1}\right)}{2}=\left(\begin{array}{ccc}
-0.008 & 0 & -0.025 \\
0 & 0.141 & 0 \\
-0.025 & 0 & -0.082
\end{array}\right)
$$

The crystallographic sets corresponding to the 24 martensitic variants possible in each austenite grain can be generated applying the cubic symmetry operations to $\varepsilon^{\mathrm{PT}}$.

The initial stress of the newly formed martensitic grain depends on the interaction between the new grain and its surroundings induced by the transformation. Here, however, the initial stress state is assumed to be equal to that of the austenitic parent grain. In addition, since the martensitic phase is much harder than the austenitic phase, we assume that it only deforms elastically. The critical resolved shear stresses of the slip systems in martensitic grains are all set to be sufficiently high to avoid plastic deformations.

\section{c) Growth of martensitic phase}

The growth of a newly formed martensite grain is described by increasing its volume fraction while decreasing the parent austenitic grain volume by the same amount. We follow an empirical law suggested by Olson and Cohen [15], who related the macroscopic strain with the macroscopic evolution of martensite, and apply it to each grain. In our model, growth of martensitic grain depends on the deformation history of its parent. The volume fraction of the

individual martensitic grain is a function of the accumulated shear strain $\Gamma=\int \sum_{\alpha}\left|\dot{\gamma}^{\alpha}\right| d t$ of its parent austenite:

$$
\frac{w^{\text {mart }}}{w_{0}^{\text {aust }}}=1-\exp \left\{-\beta[1-\exp (\alpha \Gamma)]^{n}\right\}
$$

where $\alpha, \beta$, and $n$ are the material constants; $w^{\text {mart }}$ and $w_{0}^{\text {aust }}$ are the current and initial volume weights of the martensitic grain and its parent austenitic grain, respectively.

\section{d) Implementation of MPTS}


The MPTS strain defined in b) is specific for a unit volume of martensite. As the volume of a martensitic grain increases during plastic deformation, the rate of phase transformation strain is expressed as:

$$
\dot{\boldsymbol{\varepsilon}}^{\mathrm{PT}}=\frac{\dot{w}^{\text {mart }}}{w^{\text {aust }}} \boldsymbol{\varepsilon}^{P T}
$$

The phase transformation is a source of additional plasticity accommodation in TRIP steels in addition to dislocation slip activities. The detailed treatment of MPTS in the framework of EVPSC will be discussed in the next section.

\subsection{Implementation of the PT model into EVPSC framework}

This section implements the PT model into EVPSC framework (denoted as EVPSC-PT). Detailed description of the EVPSC framework and its applications are referred to Wang et al. [23, 42-45]. With phase transformation, the total strain rate of an individual grain is the summation of elastic strain rate $\left(\dot{\boldsymbol{\varepsilon}}^{\boldsymbol{e}}\right)$, plastic strain rate $\left(\dot{\boldsymbol{\varepsilon}}^{p}\right)$ from slip systems and phase transformation strain rate ( $\dot{\boldsymbol{\varepsilon}}^{P T}$ it is zero in martensitic grains):

$$
\dot{\varepsilon}=\dot{\varepsilon}^{e}+\dot{\varepsilon}^{p}+\dot{\varepsilon}^{P T}=M^{e}: \dot{\sigma}+M^{p}: \sigma+\dot{\varepsilon}_{0}+\dot{\varepsilon}^{P T}
$$

where $\boldsymbol{M}^{\boldsymbol{e}}, \boldsymbol{M}^{\boldsymbol{p}}$ and $\dot{\boldsymbol{\varepsilon}}_{\mathbf{0}}$ are the elastic compliance, plastic compliance and the back-extrapolated strain rate, respectively. Similarly, the macroscopic strain rate $(\dot{\boldsymbol{E}})$ of the homogenous effective medium (HEM), which is the aggregate of all the grain, can be written as:

$$
\dot{E}=\bar{M}^{e}: \dot{\Sigma}+\bar{M}^{p}: \Sigma+\dot{E}_{0}
$$

where $\overline{\boldsymbol{M}}^{\boldsymbol{e}}, \overline{\boldsymbol{M}}^{\boldsymbol{p}}$ and $\dot{\boldsymbol{E}}_{\mathbf{0}}$ are the elastic compliance, plastic compliance and the back-extrapolated strain rate, respectively. Different from the single crystal law (Eq. 10), at the macroscopic level it is not possible to separate the contribution of phase transformation and the back extrapolated term to the total rate. As a consequence, only a single term $\dot{\boldsymbol{E}}_{\mathbf{0}}$ is used in Eq. 11 . The hardening of the slip systems is assumed to follow an extended Voce law, i.e.,

$$
\hat{\tau}_{\mathrm{cr}}=\tau_{0}+\left(\tau_{1}+h_{1} \Gamma\right)\left(1-\exp \left(-\frac{h_{0} \Gamma}{\tau_{1}}\right)\right)
$$


where $\tau_{0}, h_{0}, h_{1}$ and $\tau_{0}+\tau_{1}$ are the initial critical resolved shear stress (CRSS), the initial hardening rate, the asymptotic hardening rate and the back-extrapolated CRSS, respectively. The latent hardening on slip system $\alpha$ due to the activity in slip system $\beta$ is accounted for through the latent hardening coupling coefficient $h^{\alpha \beta}$ :

$$
\dot{\tau}_{c}^{\alpha}=\frac{d \hat{\tau}_{c \mathrm{r}}}{d \Gamma} \sum_{\beta} h^{\alpha \beta} \dot{\gamma}^{\beta}
$$

Here we adopt an isotropic hardening assumption: $h^{\alpha \beta}=1, \forall \alpha, \beta$.

The interaction between each grain (austenite or martensite inclusion) and the HEM is described through the elastic and the visco-plastic ellipsoidal inclusion formalism of [46]. The self-consistent condition that the macroscopic stress and strain rate must be equal to the average value over all the grains, together with the externally applied boundary conditions, provides the stress strain response of the HEM. The deformation texture is realized by updating the orientation matrices of all grains, and the internal elastic strain for comparison with the diffraction data is calculated by averaging the elastic strains for the subset of grains with the given diffraction planes along the scatter vector.

\section{Results}

The stress strain curves measured at the two different temperatures are shown in Fig. 3. For performing the EVPSC-PT modeling, the crystallographic texture of the initial austenitic phase is discretized using 23333 grains, each with a defined orientation and weight. This large number gives better peak statistics but results are nearly indistinguishable from the results obtained using 3000 orientations. The austenitic phase has the face-centered-cubic (FCC) crystallographic structure, while the martensitic phase has the body-centered-cubic (BCC) crystallographic structure. In the modeling, the single crystal elastic constants used for austenitic phase are $C_{11}=209, C_{12}=133$ and $C_{44}=121$ [47], and for martensitic phase we take the ones of iron at room temperature, i.e., $C_{11}=234, C_{12}=135$ and $C_{44}=118$ (units of GPa) [48]. The plastic deformation of the austenitic phase is assumed to be due to the $12\{111\}<110>$ slip systems, and that of martensitic phase due to the $12\{110\}<\overline{1} 11>$ slip systems. In practice, the CRSS for these latter systems is set high to eliminate the plastic contribution from martensite. The hardening parameters associated with the EVPSC-PT model are obtained by fitting the 
experimental stress strain curves at RT and $75^{\circ} \mathrm{C}$. Due to the absence of martensitic phase transformation at $75^{\circ} \mathrm{C}$, the hardening parameters associated with the austenitic phase are determined using the $75^{\circ} \mathrm{C}$ experiment and slightly adjusted empirically to describe the RT behavior during the initial $10 \%$ strain, where the volume fraction of martensitic phase is very small. The fitted hardening parameters at $75^{\circ} \mathrm{C}$ and $\mathrm{RT}$ are listed in Table 2. Note that for martensitic phase, the only non-zero hardening parameter is $\tau_{0}$, which is chosen to be sufficiently high in order to effectively eliminate plasticity in the martensite. The parameters associated with the evolution of the volume fraction of the martensitic phase are obtained by fitting the measured volume fraction of martensite $\left(\mathrm{V}^{\text {mart }}\right)$ as a function of macroscopic strain (Fig. 5). The determined parameters $\alpha, \beta$ and $n$ are also listed in Table 2. Both the stress strain curves (Figs. 4a and 4b) and the $\mathrm{V}^{\text {mart }}$-strain curve (Fig. 5) are well reproduced by the EVPSCPT model. The calculated stress strain curve that would result from excluding the martensitic transformation at RT is also included in Fig. 4a. It becomes apparent that the higher hardening rate beyond $10 \%$ strain is due the presence of martensitic transformation.

Figures 4c and 4d compare the experimental and simulated hardening rate at RT and $75^{\circ} \mathrm{C}$. The hardening rates are calculated as the derivatives of a $5^{\text {th }}$ degree polynomial fit of the stress strain curves. The hardening rate is steady at RT with increasing strain, while it decreases with strain at $75^{\circ} \mathrm{C}$. Model predicts that the hardening rate at RT also would decrease and become about half at $40 \%$ strain (Fig. 4c) without the phase transformation. As listed in Table 2, the hardening parameters of austenite at $\mathrm{RT}$ and $75^{\circ} \mathrm{C}$ are the same (except for the initial yield stress) and, therefore, the macroscopic hardening rate of this phase is the same. The EVPSC-PT model reproduces well the evolution of the hardening rate of 304 stainless steel under tension at $\mathrm{RT}$ and $75^{\circ} \mathrm{C}$. The model shows that, by comparing the hardening rate curves at RT and $75^{\circ} \mathrm{C}$, the martensitic transformation leads to increase in hardening rate beyond the strain of $10 \%$.

Figure 6 compares the deformation textures of austenitic phase at $75^{\circ} \mathrm{C}$. No martensitic phase was observed under tension at $75^{\circ} \mathrm{C}$. The EVPSC-PT model correctly predicts the deformation textures at various strain levels. As have been observed in the past for other selfconsistent polycrystal deformation models, the predicted textures are somewhat sharper, i.e. more extreme in the high and low areas, than the measured textures, but the spatial locations of the high and low points are well matched. 
Figure 7 shows the experimental and simulated textures of austenitic and martensitic phases under tension at RT. The predicted deformation textures of austenitic phase agree well with the experimental ones, which also suggests that the occurrence of martensitic phase does not significantly change the texture development in the austenitic phase. The predicted textures of the martensitic phase agree well with the experimental textures, especially at higher volume fractions of the martensitic phase. At $40 \%$ strain the main texture components are well captured by the EVPSC-PT model. Overall, the good agreement between model-predicted and experimental textures supports the variant selection criterion and the austenite-to-martensite rotation matrix used here.

The experimental and simulated peak intensities $\left(I_{L}^{h k l}\right)$ of various diffraction families are shown in Fig. 8 and follow from integrating the orientation distributions shown in Figs. 6 and 7 over a solid angle of $14^{\circ}$ defined by the neutron detector. The peak intensity is represented as the multiple of random (MRD) values, defined as the ratio of the volume fraction of the grains with the same diffraction planes in the current texture and in a random distribution of orientations, for each phase. Before doing this, the volume fraction of each phase is normalized to 1 . As can be seen, the evolution of $\{111\}$ diffraction peak intensities of austenite follows the experimental trend, i.e. an increase followed by a decrease, although the model over-predicts such evolution as a consequence of over-predicting the $\{111\}$ component along the RD (Fig 7). The $\{200\}$ and \{311\} peak intensities of austenite remain almost constant in the experiment and the model captures this behavior.

For the martensite the experimental intensities of $\{310\}$ and $\{321\}$ diffraction planes increase with stress, while those of $\{110\}$ and $\{211\}$ decrease from initially high values. The predicted peak intensity evolution, on the other hand, is nearly constant for $\{211\},\{310\},\{321\}$ and $\{110\}$ intensities and differs significantly from the experiment, especially at stress levels lower than $900 \mathrm{MPa}$. At stresses above $900 \mathrm{MPa}$, (i.e., martensite volume fraction higher than 0.15), the model seems to capture the experimental evidence, a feature that hints at what may be lacking in the model and which will be discussed in connection with Fig. 9.

The measured and simulated evolution of the internal elastic strain of various diffraction planes is plotted in Fig. 9. At $75^{\circ} \mathrm{C}$, the internal elastic strains of $\{111\},\{311\}$ and $\{200\}$ diffraction planes in austenite are correctly predicted by the EVPSC model, except for the 
saturation trend of the $\{111\}$ strain at stresses higher than $800 \mathrm{MPa}$ (Fig. 9c). The latter may be attributed to the larger predicted $\{111\}$ texture component along the loading direction. Without phase transformation the internal elastic strains in austenite at RT are the same as for $75^{\circ} \mathrm{C}$, and do not capture load transfers at applied stresses larger than $800 \mathrm{MPa}$, a point which can be correlated with the appearance of the martensitic phase. The predictions are significantly improved, trend-wise, by accounting for the martensitic transformation.

The comparison of predicted and measured internal elastic strain evolution in the martensitic phase (Fig. 9b) shows large discrepancies, the origin of which may be attributed to the following. The martensite forms as inclusions inside the austenitic phase, and the shear and dilatation components of the transformation strain are expected to induce a large reaction stress upon the martensite, conceptually similar to the one induced by deformation twinning transformation in HCP as discussed by [49]. Since we have no information about such back stresses, in this work we used the simpler (and clearly insufficient) assumption that the initial nucleation stress state in the martensitic grain is equal to the current stress in its parent austenitic grain. The fact that the measured elastic lattice strain of $\{211\},\{311\}$ and $\{310\}$ families increase rapidly with further loading may be attributed to an erase of the initial reaction stress in the martensite as the applied stress and the martensite volume increase. The same explanation may be used for the rapid decrease in internal strain associated with the $\{110\}$ family (Fig 9b): $\{110\}$ is approximately the habit plane of martensite along which the dilatational component associated with MPTS is aligned, and thus may be inducing a compressive reaction from the austenite matrix. To improve the predictability of the model, a correct estimate of the back stress induced by the formation of martensite needs to be implemented in the model when the martensite lamella is created. Such a calculation would involve performing local deformation simulation of the transformation, as was done by Arul Kumar et al [49] for twinning transformations using Crystal Plasticity Fast Fourier Transformation (CPFFT). Such calculation is outside the scope of this work and will be tackled in the future. In addition, in the model the martensite volume fraction is an explicit function of the accumulated shear strain in the grain via the empirical law of Olson-Cohen (Eq. 8), independently of the grain stress. However, a stressdriven mechanism for driving martensitic transformation should be more appropriate. A model including a more sophisticated treatment of stress-driven nucleation and incorporation of the induced reaction stress is in progress and will be reported in future work. 
Figure 10 shows the contribution of the elastic strain, plastic strain, strain from slip and strain from phase transformation to the total strain. Although the contribution of the phase transformation to the total strain is relatively small, it influences the behavior of the 304 stainless steel significantly

\section{Discussion}

The effect of martensitic phase transformation on macroscopic stress strain response, texture evolution and internal elastic strain evolution at room temperature is systematically investigated by in-situ neutron diffraction, electron backscatter diffraction and crystal plasticity modeling in 304 stainless steel. The proposed phase transformation model correctly predicts the stress strain response, phase fraction evolution, and texture evolution. The model also captures the general trend of the evolution of internal elastic strain and diffracted intensities in the austenitic phase, but it has some limitations in what concerns equivalent magnitudes in the martensite.

The experimental EBSD evidence of our work reveals that usually one martensite variant - formed by finely and evenly distributed domains inside the grains - is prevalent in individual grains. In addition, simultaneously measured stress strain response and martensitic phase fraction evolution reveals that the 304 stainless steel deforms without appreciable phase transformation at $75^{\circ} \mathrm{C}$ and with substantial phase transformation at room temperature. The modeling interpretation supports the conclusion that the presence of the martensitic phase increases substantially the hardening rate, although it makes little contribution to the overall deformation. In addition, the appearance of martensite can be correlated with a clear load transfer into the austenite, as evidenced by comparing Figs. 9a and 9c.

In the model, the evolution of the martensitic phase is governed by an empirical law based on the accumulated shear strain in each grain (Eq. 7). While this law is fitted to the measured evolution of the total martensitic phase fraction (Fig. 5), its functional dependence lacks a physical basis and we suspects that it is partially responsible for the discrepancies between experimental and predicted evolution of the internal elastic lattice strain in the martensite. A stress driven nucleation criterion should change the rate at which martensite 
transforms and, in addition to being physically more appealing, it should also affect the predicted evolution of internal strain in grains.

Once the 'strain based' criterion for allowing transformation is met, an elastic-energybased variant selection criterion is employed to choose which of the 24 martensitic variants is created. Because the texture evolution of martensitic phase is very sensitive to the variant selection criterion, and because predicted martensite texture components are consistent with the experimental, we believe that this energy-based variant selection criterion is appropriate. The fact that there are some differences in the early stages of transformation suggest that the criterion can be improved, possibly by allowing more than one martensitic variant to be activated in each grain. A model incorporating more physics-based criteria for driving martensite transformation and accounting for back stress effects on austenite and martensite following martensite nucleation should improve the predictive capabilities and will be the focus of our future work.

\section{Conclusions}

The effect of martensitic phase transformation on macroscopic stress strain relation, texture evolution and internal elastic strain evolution at room temperature is systematically investigated by in-situ neutron diffraction, electron backscatter diffraction and crystal plasticity modeling. The following conclusions can be drawn:

1. The EBSD orientation map reveals that mostly a single martensite variant is formed in individual grains of 304 stainless steel. The martensite is finely and evenly distributed inside the grains.

2. Comparing to the same 304 stainless steel under deformation without phase transformation at $75^{\circ} \mathrm{C}$, the significant hardening rate increase of the material at room temperature is ascribed entirely to the formation of martensitic phase.

3. A phase transformation crystal plasticity model is proposed that correctly predicts the texture evolution of martensite and austenite phase, so indicating that our criterion for choosing martensite variants is appropriate. The model also captures the general trend in the evolution of internal elastic strain and diffracted intensities for the austenite phase. The poor agreement obtained for the prediction of the same magnitudes in the martensite phase suggests that transformation induced initial stresses play an important role and need to be accounted for with improved lower scale mechanical models. 


\section{Acknowledgment}

This work is funded by the U.S. Dept. of Energy, Office of Basic Energy Sciences Project FWP 06SCPE401. This work has benefited from the use of SMARTS and HIPPO at the Lujan Center at Los Alamos Science Center. Los Alamos National Laboratory is operated by Los Alamos National Security LLC under DOE contract DE-AC52-06NA25396.

\section{References}

[1] Matsumura O, Sakuma Y, Takechi H. Trans Iron Steel Ins Japan 1987;27:570.

[2] Bleck W, Schael I, Steel Res 2000;71:173.

[3] Jacques PJ, Furnemont Q, Lani F, Pardoen T, Delannay F. Acta Mater 2007;55:3681.

[4] Patel JR, Cohen M. Acta Metall 1953;1:531.

[5] Angel T. J Iron Steel Ins 1954;177:165.

[6] De Cooman BC. Curr Opin Solid State Mater Sci 2004;8:285.

[7] Jacques PJ, Furnemont Q, Godet S, Pardoen T,Conlon KT, Delannay F. Philos Mag 2006;86:2371.

[8] Greenwood GW, Johnson RH. Proc R Soc London 1965;A283:403.

[9] Leblond JB, Mottet G, Devaux JC. J Mech Phys Solids 1986;34:395.

[10] Leblond JB, Mottet G, Devaux JC. J Mech Phys Solids 1986;34:411.

[11] Gibbs PJ, De Cooman BC, Brown DW, Clausen B, Schroth JG, Merwin MJ, Matlock DK. Mater Sci Eng, A 2014;609:323.

[12] Oliver EC, Mori T, Daymond MR, Withers PJ. Acta Mater 2003;51:6453.

[13] An K, Skorpenske HD, Stoica AD, Ma D, Wang XL, Cakmak E. Metall Mater Trans A 2011;42:95.

[14] Wang H, Clausen B, Tomé CN, Wu PD. Acta Mater 2013;61:1179.

[15] Olson GB, Cohen M. Metall Trans A 1975;6:791.

[16] Bhattacharyya A, Weng GJ. J Mech Phys Solids 1994;42:1699.

[17] Cherkaoui M, Berveiller M, Sabar H. Int J Plast 1998;14:597.

[18] Cherkaoui M, Berveiller M, Lemoine X. Int J Plast 2000;16:1215.

[19] Turteltaub S, Suiker ASJ. J Mech Phys Solids 2005;53:1747.

[20] Turteltaub S, Suiker ASJ, Int J Solids Struct 2006;43:4509.

[21] Delannay L, Jacques PJ, Pardoen T. Int J Solids Struct 2007;45:1825.

[22] Wang H, Raeisinia B, Wu PD, Agnew SR, Tomé CN. Int J Solids Struct 2010;47:2905.

[23] Wang H, Wu PD, Tomé CN, Huang Y. J Mech Phys Solids 2010;58:594.

[24] Wang H, Wu PD, Tomé CN, Wang J. Mater Sci Eng, A 2012;555:93.

[25] Wang H, Wu PD, Wang J, Tomé CN, Int J Plast 2013;49:36.

[26] Wang H, Wu PD, Wang J. Int J Plast 2013;47:49.

[27] Guo XQ, Wu PD, Wang H, Mao XB. Steel Res Int 2015;86:894.

[28] Wang H, Wu PD, Tomé CN, Wang J. Int J Solids Struct 2012;49:2155.

[29] Lee SY, Wang H, Gharghouri MA, Nayyeri G, Woo W, Shin E, Wu PD, Poole WJ, Wu W, An K. Acta Mater 2014;73:139.

[30] Wang H, Clausen B, Capolungo L, Beyerlein IJ, Wang J, Tomé CN. Int J Plast 2015;in press. doi: 10.1016/j.ijplas.2015.07.004.

[31] Qiao H, Wu PD, Wang H, Gharghouri MA, Daymond MR. 2015;71:308. 
[32] Bourke MAM, Dunand DC, Ustundag E. Applied Physics A 2002;74(Suppl):S1707.

[33] Han HN, Suh DW. Acta Mater 2003;51:4907.

[34] Gey N, Petit B, Humbert M. Metall Mater Trans A 2005;36:3291.

[35] Wechsler MS, Lieberman DS, Read TA. Trans. AIME J Met 1953;197:1503.

[36] Bowles JS, Mackenzie JK. Acta Metall 1954;2:129.

[37] Mackenzie JK, Bowles JS. Acta Metall 1954;2:138.

[38] Kurdjumov GV, Sachs G. Z Physik 1930;64:325.

[39] Nishiyama Z. Sci Rep Tohoku Imp Univ Tokio 1934;23:637.

[40] Bhadeshia HKDH. Worked examples in the geometry of crystals. The Institute of Metals North American Publications Center. 2001.

[41] Dyson DJ, Holmes B. J Iron Steel Ins 1970;208:469.

[42] Wang H, Wu PD, Boyle KP, Neale KW. Int J Solids Struct 2011;48:1000.

[43] Wang H, Wu PD, Wang J. Comput Mater Sci 2015;96:214.

[44] Wang H, Wu PD, Lee SY, Wang J, Neale KW. Int J Mech Sci 2015;92:70.

[45] Wang H, Wu PD, Wang J. Metall Mater Trans A 2015;46,3079.

[46] Eshelby JD. Proc Royal Soc Lond A 1957;241:376.

[47] Ledbetter HM. Physica B+C 1985; 128:1.

[48] Simmons G, Wang H. 1971. Single Crystal Elastic Constants and Calculated Polycrystal Properties. MIT Press, Cambridge (MA), 137.

[49] Arul Kumar M, Kanjarla AK, Niezgoda SR, Lebensohn RA, Tomé CN. Acta Mater 2015;84:349. 


\section{Table titles:}

Table 1. Chemical composition of the stainless steel in weight percent.

Table 2. The hardening parameters for Voce hardening law for austenite and martensite phases studied in this work.

\section{Figure captions:}

Figure 1. EBSD orientation map of 304 stainless steel sheet at tensile strains of (a) $0 \%$ (undeformed) and (b) 30\%.

Figure 2. $\{111\},\{200\}$ and $\{220\}$ pole figures for initial texture.

Figure 3. True stress strain curves measured at RT and $75^{\circ} \mathrm{C}$ during in-situ neutron diffraction and uninterrupted loading at $10^{-5} \mathrm{~s}^{-1}$. The solid lines correspond to instantaneous readings from extensometer and load cell. The symbols correspond to values averaged during 8 minute intervals corresponding to neutron diffraction counts.

Figure 4. Experimental and simulated stress strain curves of 304 stainless steel under tension at (a) RT and (b) $75^{\circ} \mathrm{C}$, and the corresponding hardening rates at (c) RT and (d) $75^{\circ} \mathrm{C}$.

Figure 5. Experimental and simulated $V^{\text {mart }}$-strain curves of 304 stainless steel under tension at RT.

Figure 6. Experimental and simulated textures of austenitic phase of 304 stainless steel under tension at $75^{\circ} \mathrm{C}$.

Figure 7. Experimental and simulated textures of austenitic and martensitic phases of 304 stainless steel under tension at RT.

Figure 8. Experimental and predicted diffraction peak intensities defined with respect to a random distribution for planes perpendicular to the tensile axis of (a) austenitic and (b) martensitic phases at RT.

Figure 9. Experimental and simulated internal elastic strain of various diffraction planes in (a) austenitic phase ( $\gamma$ phase) at RT, (b) martensitic phase ( $\alpha$ phase) at RT, and (c) austenitic phase ( $\gamma$ phase) at $75^{\circ} \mathrm{C}$.

Figure 10. Contribution of the elastic strain, strain from slip and strain from phase transformation to the total strain. 
Table 1. Chemical composition of the stainless steel in weight percent.

\begin{tabular}{|c|c|c|c|c|c|c|}
\hline Element & $\mathrm{C}$ & $\mathrm{Cr}$ & $\mathrm{Ni}$ & $\mathrm{Si}$ & $\mathrm{Mn}$ & $\mathrm{Mo}$ \\
\hline Fraction & 0.08 & 19.0 & 9.25 & 0.75 & 2.0 & 0 \\
\hline
\end{tabular}

Table 2. The hardening parameters for Voce hardening law for austenite and martensite phases studied in this work.

\begin{tabular}{|c|c|c|c|c|c|c|c|c|}
\hline & \multicolumn{4}{|c|}{ austenite } & \multicolumn{3}{c|}{ martensite } \\
\hline & $\tau_{0}(\mathrm{MPa})$ & $\tau_{1}(\mathrm{MPa})$ & $\mathrm{h}_{0}(\mathrm{MPa})$ & $\mathrm{h}_{1}(\mathrm{MPa})$ & $\tau_{0}(\mathrm{MPa})$ & $\alpha$ & $\beta$ & $\mathrm{n}$ \\
\hline $75^{\circ} \mathrm{C}$ & 100 & 96 & 550 & 190 & $\mathrm{NA}$ & NA & NA & NA \\
\hline $\mathrm{RT}$ & 110 & 96 & 550 & 190 & 1600 & 0.18 & 11.8 & 2.2 \\
\hline
\end{tabular}
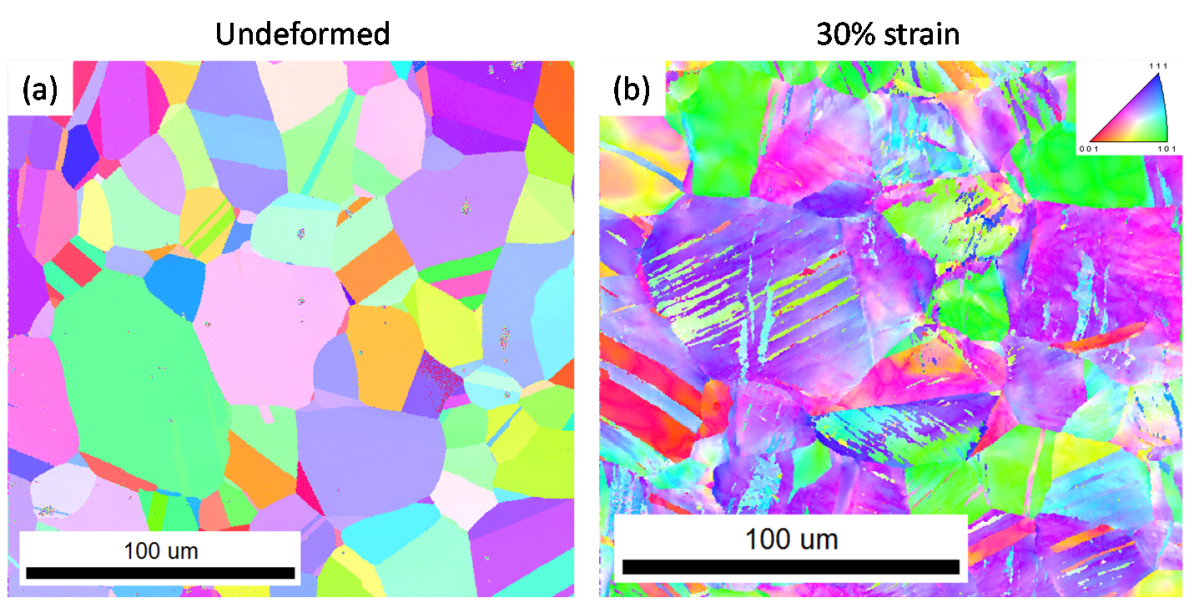

Figure 1. EBSD orientation map of 304 stainless steel sheet at tensile strains of (a) $0 \%$ (undeformed) and (b) $30 \%$.

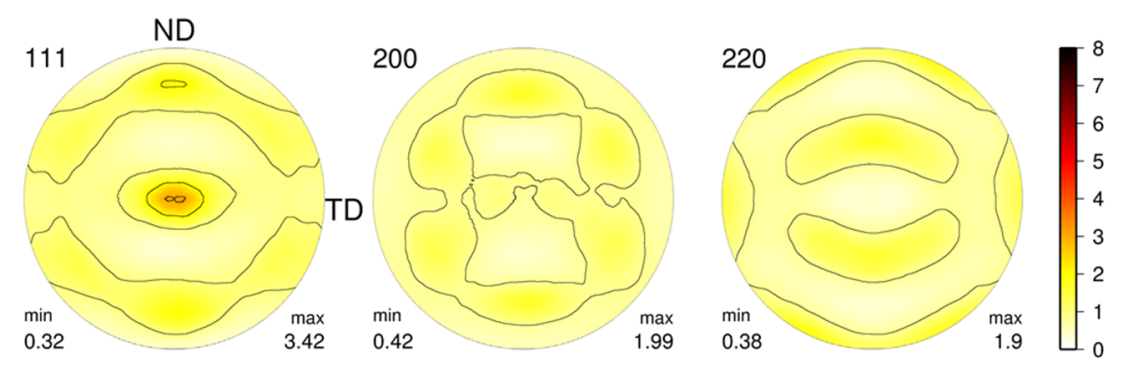

Figure 2. $\{111\},\{200\}$ and $\{220\}$ pole figures for initial texture. 


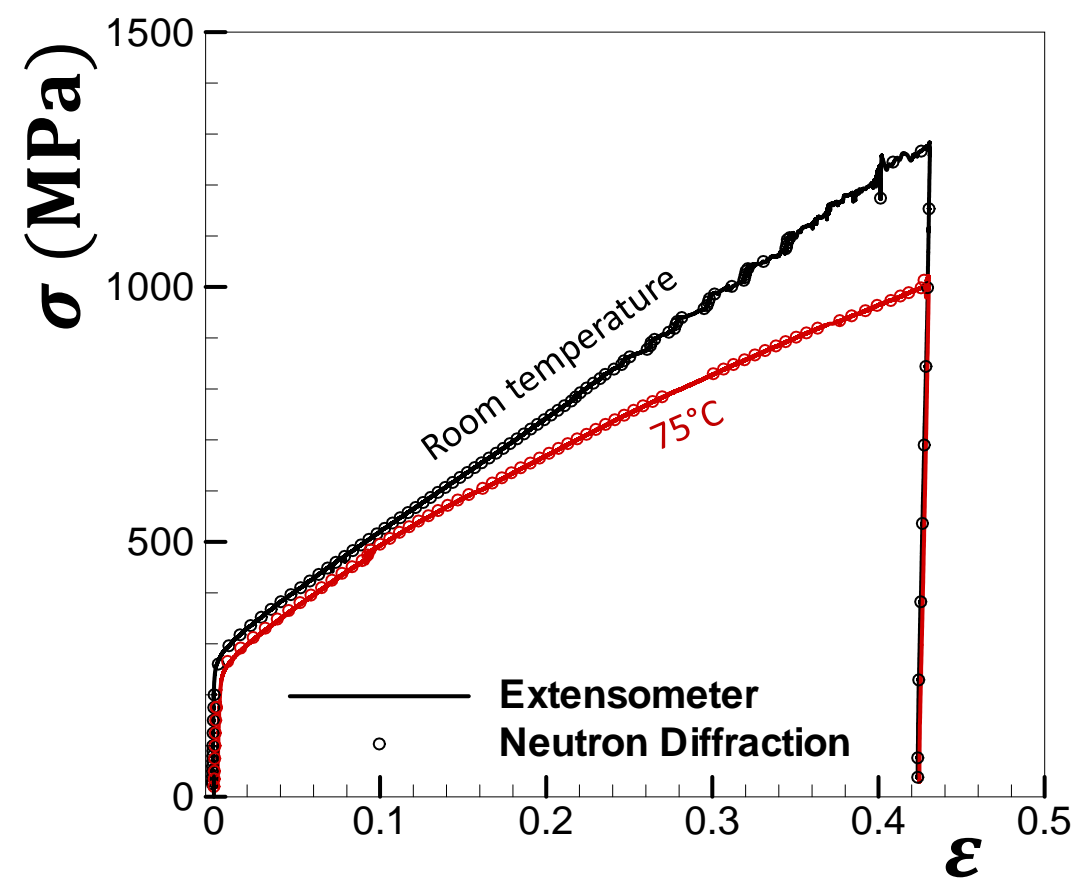

Figure 3. True stress strain curves measured at RT and $75^{\circ} \mathrm{C}$ during in-situ neutron diffraction and uninterrupted loading at $10^{-5} \mathrm{~s}^{-1}$. The solid lines correspond to instantaneous readings from extensometer and load cell. The symbols correspond to values averaged during 8 minute intervals corresponding to neutron diffraction counts. 

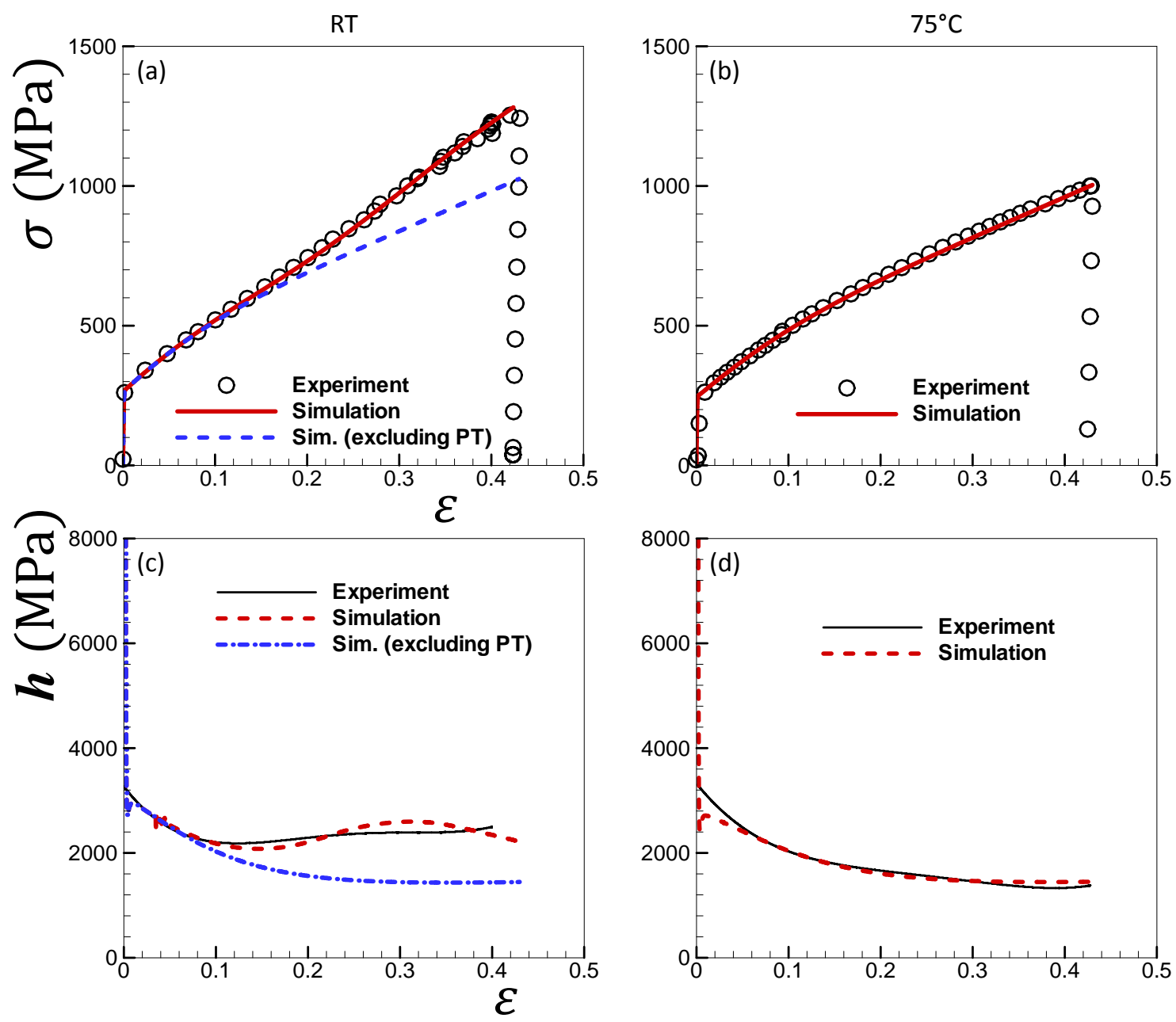

Figure 4. Experimental and simulated stress strain curves of 304 stainless steel under tension at (a) RT and (b) $75^{\circ} \mathrm{C}$, and the corresponding hardening rates at (c) RT and (d) $75^{\circ} \mathrm{C}$.

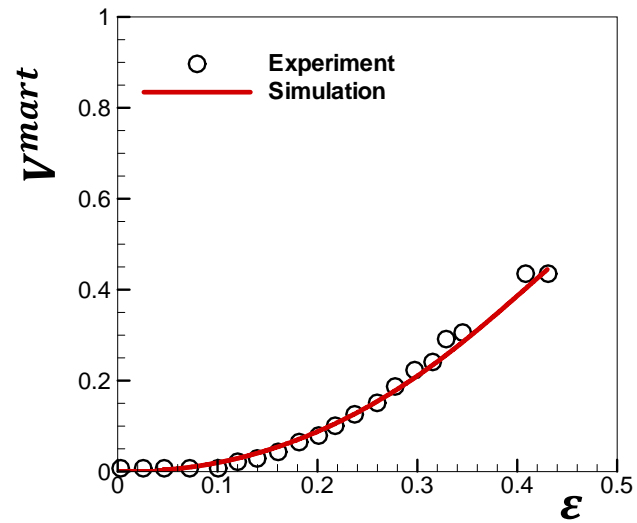

Figure 5. Experimental and simulated $V^{\text {mart }}$-strain curves of 304 stainless steel under tension at RT. 


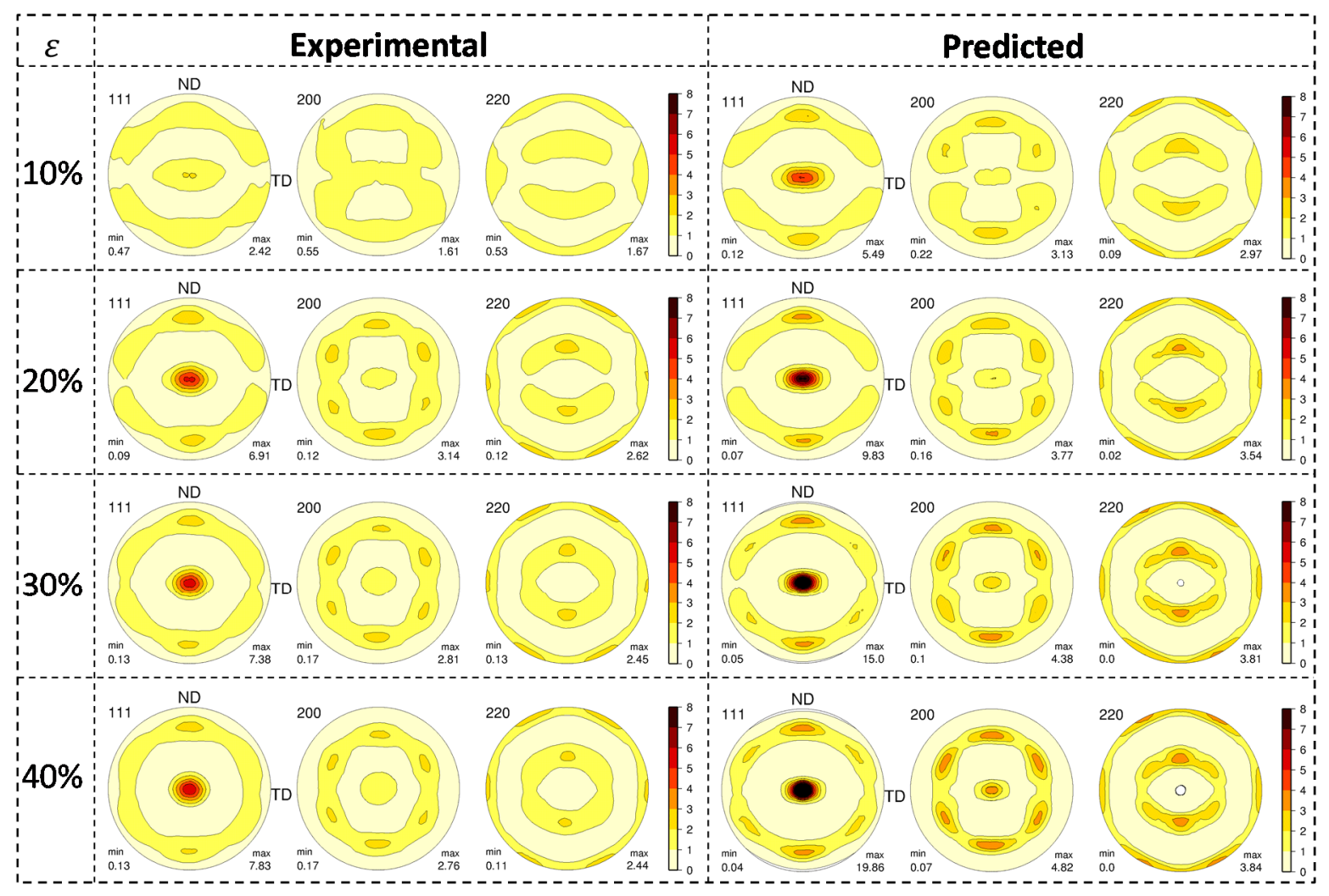

Figure 6. Experimental and simulated textures of austenitic phase of 304 stainless steel under tension at $75^{\circ} \mathrm{C}$. 


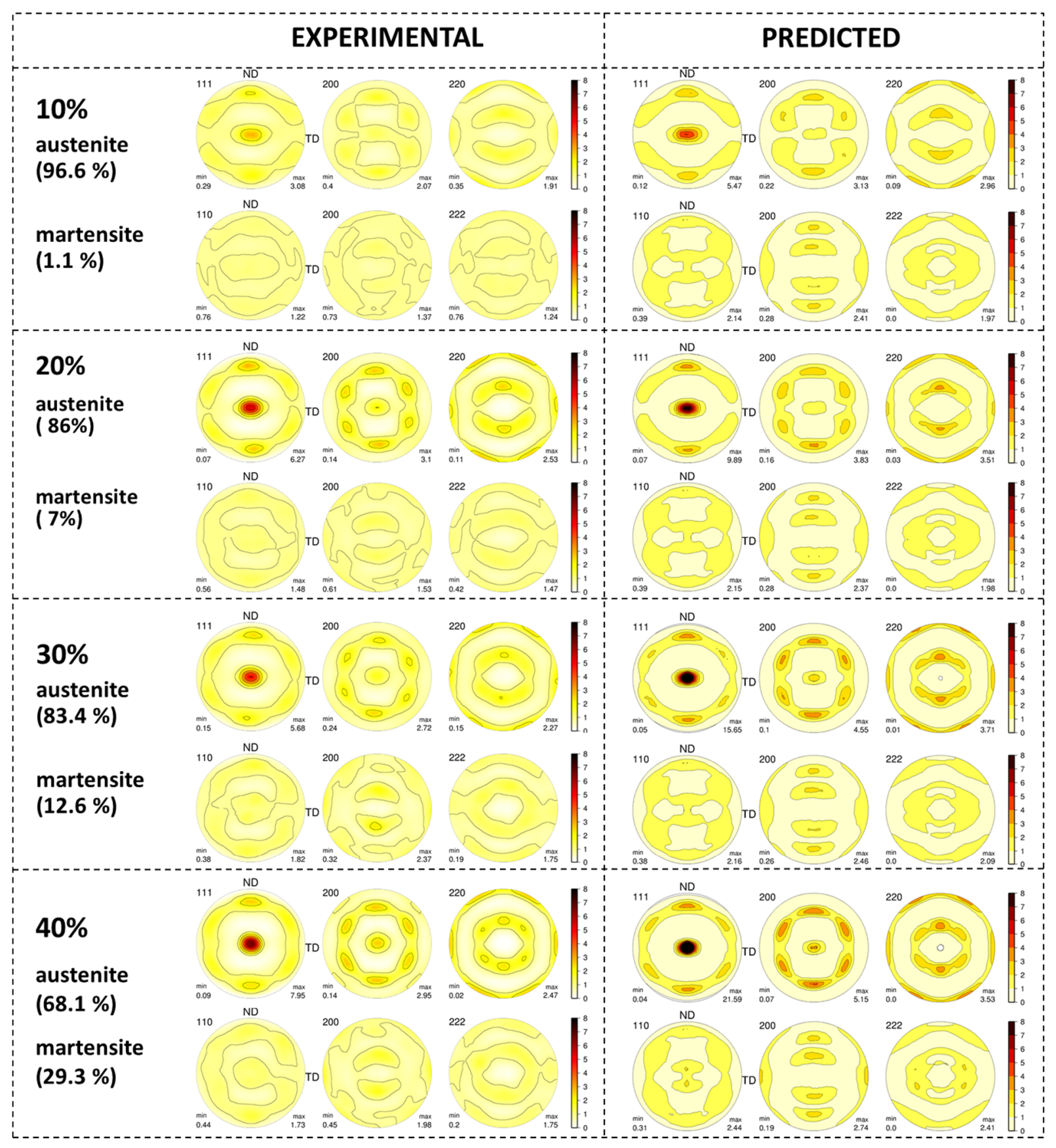

Figure 7. Experimental and simulated textures of austenitic and martensitic phases of 304 stainless steel under tension at RT. 

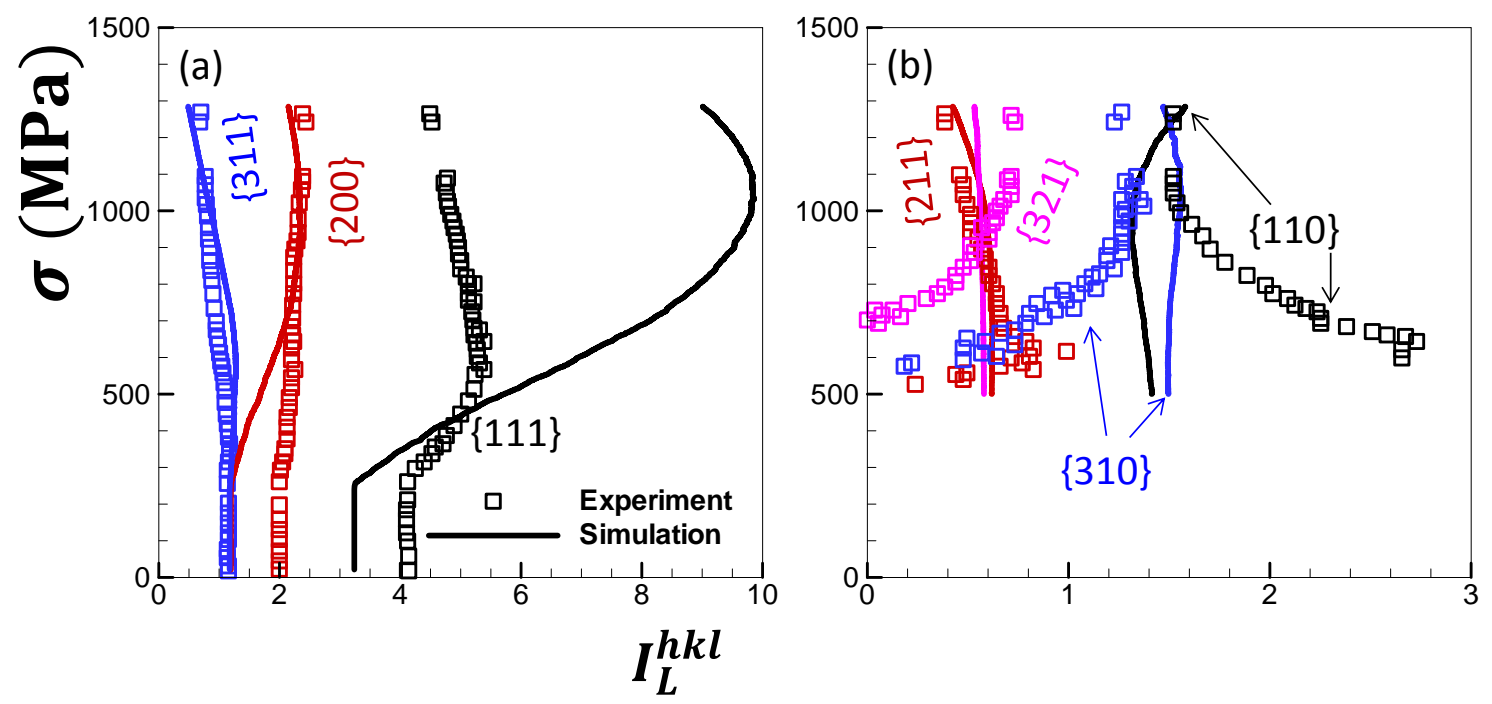

Figure 8. Experimental and predicted diffraction peak intensities defined with respect to a random distribution for planes perpendicular to the tensile axis of (a) austenitic and (b) martensitic phases at RT. 

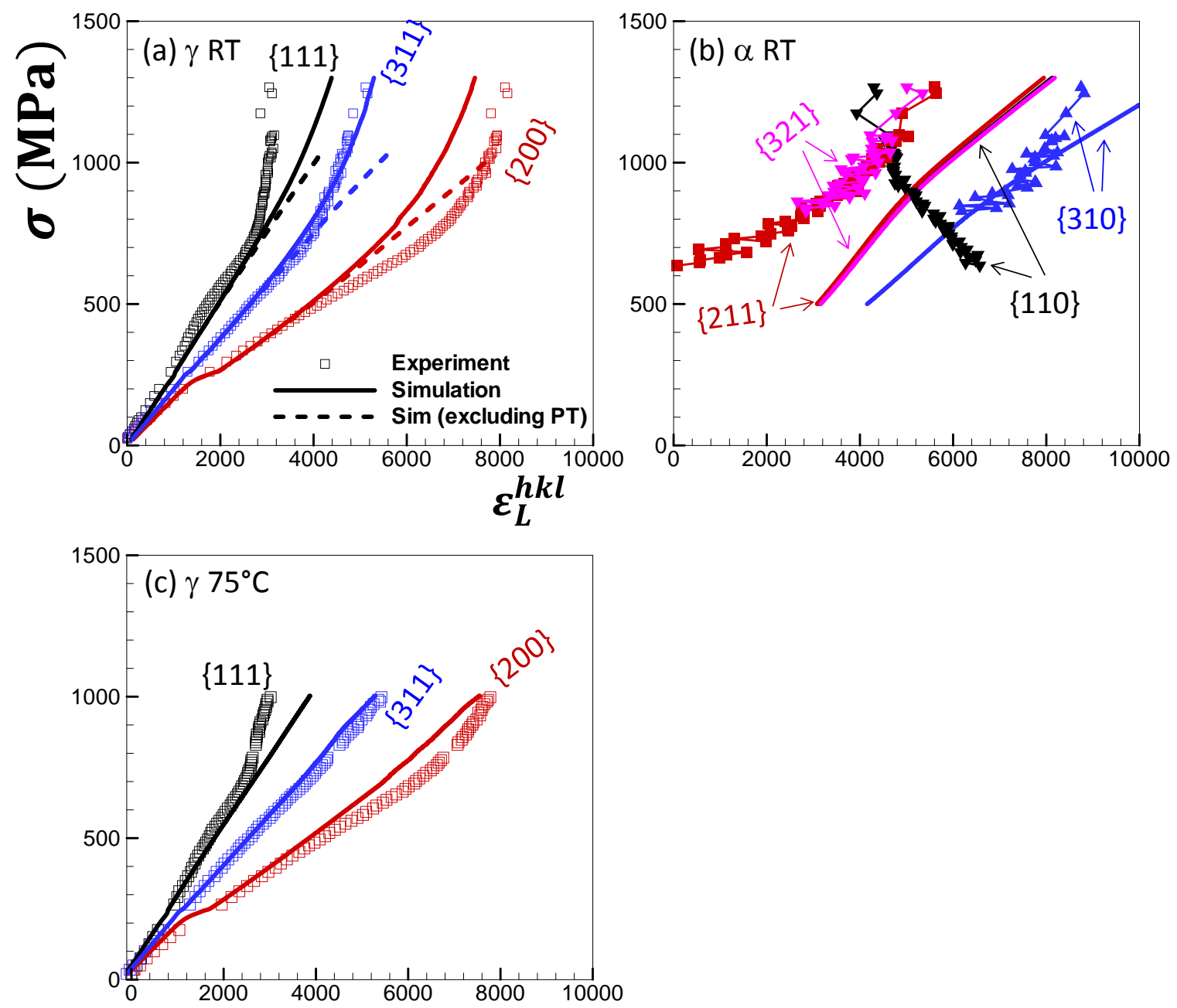

Figure 9. Experimental and simulated internal elastic strain of various diffraction planes in (a) austenitic phase ( $\gamma$ phase) at RT, (b) martensitic phase ( $\alpha$ phase) at RT, and (c) austenitic phase ( $\gamma$ phase) at $75^{\circ} \mathrm{C}$. 


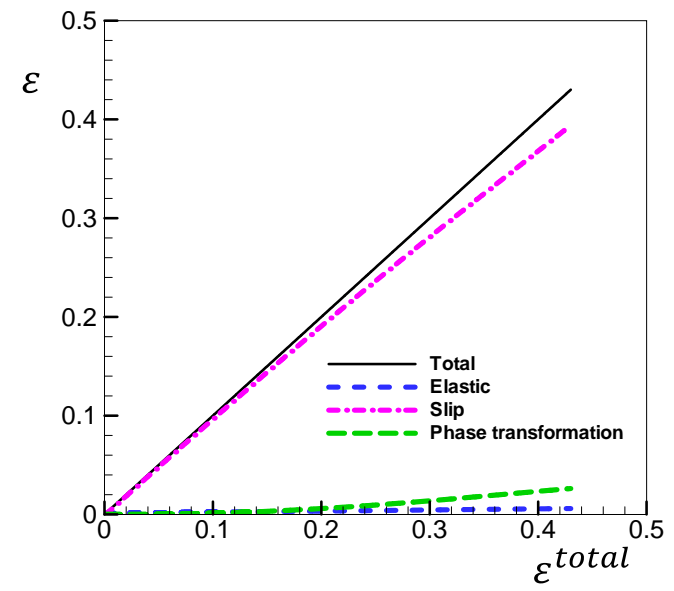

Figure 10. Contribution of the elastic strain, strain from slip and strain from phase transformation to the total strain. 